\title{
Óleo de copaíba (Copaifera sp.): histórico, extração, aplicações industriais e propriedades medicinais
}

\author{
PIERI, F.A. ${ }^{1 *}$; MUSSI, M.C. ${ }^{2}$; MOREIRA, M.A.S. ${ }^{1}$ \\ 'Universidade Federal de Viçosa, Departamento de Veterinária, Setor de Medicina Veterinária Preventiva e Saúde \\ Pública, CEP: 36570-000, Viçosa-Brasil *fabio.pieri@ufv.br ${ }^{2}$ Universidade de São Paulo, Departamento de \\ Estomatologia, CEP: 17012-901, Bauru-Brasil.
}

\begin{abstract}
RESUMO: Este trabalho teve como objetivo realizar uma revisão de literatura sobre o gênero Copaifera sp. Foi realizado levantamento bibliográfico do período de 1792 a 2008 utilizando bibliotecas da Universidade de São Paulo, Universidade Federal de Viçosa, Universidade Federal de Alfenas e Universidade José do Rosário Vellano, pesquisas às bases de dados SCOPUS e PubMed, além de ferramentas de busca na web. Utilizou-se para a busca palavras chave como "Copaiba", "Copaifera", "Copaíba oil" "Óleo de Copaíba". Como resultado desta pesquisa obtevese a seleção de 63 referências incluindo livros, artigos, cadernos técnicos, resumos de congressos, teses, dissertações e patentes. Estes dados apontaram o óleo de copaíba como um exsudato produzido pelas copaibeiras como defesa contra seus predadores, que vem sendo utilizado pela medicina tradicional popular e silvícola há mais de 500 anos. Ele é extraído destas árvores através de perfurações realizadas em seus troncos. Além das inúmeras aplicações do óleo em cosméticos e outras indústrias, ainda há uma série de indicações para seu uso na medicina. Existem hoje descritas algumas dezenas de propriedades medicinais diferentes, que vem sendo em alguns casos comprovadas cientificamente, como atividade antimicrobiana, antiinflamatória, anti-neoplásica entre outras. Estudos recentes têm demonstrado também grande potencial de uso do óleo de copaíba na odontologia, na composição de cimentos endodônticos e na prevenção e combate da doença periodontal. As informações contidas neste trabalho demonstram uma grande variabilidade de aplicações do óleo de copaíba. Entretanto uma quantidade limitada de pesquisas sobre suas propriedades medicinais tem sido realizada, apresentando assim a necessidade de novas pesquisas sobre estas.
\end{abstract}

Palavras-chave: plantas medicinais, medicamentos fitoterápicos, Copaifera, óleo de copaíba

\begin{abstract}
Copaiba oil (Copaifera sp.): history, extraction, industrial applications and medicinal properties. The aim of this study was to conduct a literature review concerning the genus Copaifera sp. The survey involved the period from 1792 to 2008 and was done in the libraries of University of São Paulo, Federal University of Viçosa, Federal University of Alfenas and University Jose do Rosario Vellano, using SCOPUS and PubMed databases, and web search engines. The words "Copaiba", "Copaifera," "Óleo de copaiba" and "Copaiba oil" were used. This research resulted in the selection of 63 references including books, articles, technical specifications, conference abstracts, theses, dissertations and patents. These data indicate copaiba oil is an exudate from copaiba trees, produced as a defense against their predators and that has been used in traditional, popular and forestry medicine for over 500 years. It is extracted from trees through holes made in their trunks. Besides the oil applications in cosmetic and other industries, there are still a number of indications for its use in medicine. So far, dozens of different medicinal properties have been described and, in some cases, scientifically proven, including antimicrobial, anti-inflammatory, and anti-cancer activity. Recent studies have also shown great potential for the use of copaiba oil in dentistry like in endodontic cement composition, as well as in the prevention and therapy of periodontal diseases. This work showed a great variety of applications to copaiba oil. However, few studies on its medicinal properties have been performed, showing thus the need for further research.
\end{abstract}

Key words: medicinal plants, phytotherapic drugs, Copaifera, copaiba oil

Recebido para publicação em 25/09/2008

Aceito para publicação em 23/04/2009

Rev. Bras. Pl. Med., Botucatu, v.11, n.4, p.465-472, 2009. 


\section{A COPAÍBA}

As copaibeiras (Figura 1) são árvores comuns à América Latina e África Ocidental (Francisco, 2005), sendo encontradas, no Brasil, nas regiões Sudeste, Centro-Oeste e Amazônica. Tais plantas chegam a viver cerca de 400 anos, atingem altura entre 25 e 40 metros (Araújo Júnior et al., 2005), diâmetro entre 0,4 e 4 metros, possuem casca aromática, folhagem densa, flores pequenas e frutos secos, do tipo vagem. As sementes são pretas e ovóides com um arilo amarelo rico em lipídeos (Alencar, 1982; Van Den Berg, 1982; Lorenzi, 1992; Xena \& Berry, 1998).

Da árvore da copaíba é extraído um óleoresina, de cor que varia de amarelo ouro a marrom (Lloyd, 1898), dependendo da espécie. Esse óleoresina tem sido utilizado desde a época da chegada dos portugueses ao Brasil na medicina tradicional popular e silvícola para diversas finalidades, e hoje se encontra como um dos mais importantes produtos naturais amazônicos comercializados, sendo também exportado para Estados Unidos, França, Alemanha e Inglaterra (Veiga Junior \& Pinto, 2002).

Há também grande interesse na madeira de algumas espécies de copaíba, pela sua superfície lisa, lustrosa, durável, de alta resistência ao ataque de xilófagos e baixa permeabilidade, que são características desejáveis para o uso na fabricação de peças torneadas e para a marcenaria em geral (Carvalho, 1942). Aárvore também tem sido utilizada para a fabricação de carvão (Loureiro, 1979) e pelas indústrias de construção civil e naval (Carvalho, 1994; Veiga Junior \& Pinto, 2002).

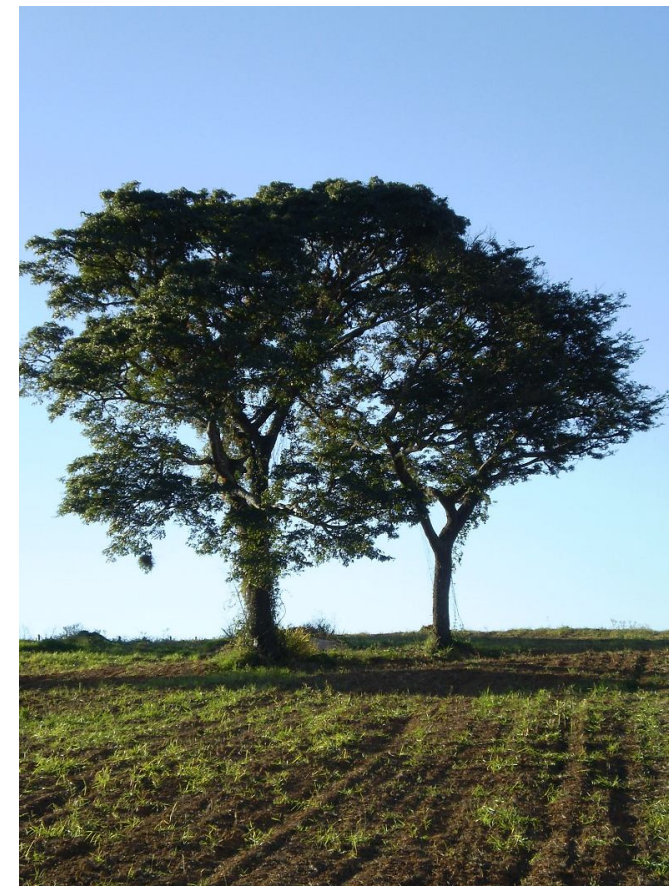

FIGURA 1. Árvores de copaíba (Copaifera langsdorffii)
No Brasil, as árvores são conhecidas como copaíba, copaibeira, pau-de-óleo, copaúva, copai (Cascon, 2004), copaibarana (Rodrigues, 1989), copaibo, copal, marimari e bálsamo dos jesuítas, e o óleo é chamado de óleo de copaíba ou bálsamo. Nos demais países da América Latina, denomina-se a árvore como "palo-de-bálsamo", "aceite", "cabima", entre outros. A denominação do óleo-resina em inglês é "copaíba balsam" e do óleo volátil, "copaíba oil". Em francês a árvore é denominada "copayer", a óleoresina, "baume de copayer" e o volátil, "huile de copayer" (Cascon, 2004).

Essa planta se apresenta na classificação botânica, pertencente à família Leguminosae, subfamília Caesalpinoideae, gênero Copaifera, (Lloyd, 1898; Brito et al., 2000; Maciel et al., 2002; Cascon, 2004; Veiga Junior et al., 2005; Oliveira et al., 2006) e, segundo o Index Kewensis (1996), possui 72 espécies descritas, sendo 16 delas encontradas exclusivamente no Brasil (Veiga Junior \& Pinto, 2002).

Entre as espécies mais abundantes no Brasil e América do Sul estão a Copaifera officinalis L. (Norte do Amazonas, Roraima, Colômbia e Venezuela), a Copaifera guianensis (Guianas), Copaifera reticulata Ducke, Copaifera multijuga Hayne (Amazônia), Copaifera confertiflora (Piauí), Copaifera langsdorffii (Brasil, Argentina e Paraguai), Copaifera cariacea (Bahia) e Copaifera cearensis Huber ex Ducke (Ceará) (Wood et al., 1940; Mors \& Rizzini, 1966).

No continente Africano, na região do Congo, Camarões, Guiné e Angola, são descritas cerca de 19 espécies de copaíba. Nessas regiões é comum a presença de citações de âmbares (óleo-resinas fossilizadas) provenientes de espécies de Copaifera. Há também uma espécie descrita na ilha de Bornéu na Malásia, chamada Copaífera palustris, a qual se assemelha muito às espécies africanas (Veiga Junior \& Pinto, 2002).

O objetivo deste trabalho foi fazer uma revisão de literatura, apresentando esta árvore nativa de nosso território, apontando os diversos usos ao longo da história na medicina popular e silvícola a mais de 500 anos e o surgimento, ainda que modesto, de algumas pesquisas sobre as propriedades medicinais.

Para tanto, foi realizado um levantamento bibliográfico do período de 1792 a 2008 utilizando bibliotecas da Universidade de São Paulo, Universidade Federal de Viçosa, Universidade Federal de Alfenas e Universidade José do Rosário Vellano, pesquisas às bases de dados SCOPUS e PubMed, além de ferramentas de busca na web. Utilizou-se para a busca palavras chave como "Copaiba", "Copaifera", "Copaíba oil” "Óleo de Copaíba".

Como resultado desta pesquisa obteve-se a seleção de 63 referências incluindo 28 livros, 21 artigos, 5 cadernos técnicos, 3 resumos em anais de congressos, 3 teses de doutorado, 1 dissertação 
de mestrado e 2 patentes, que foram utilizadas para a confecção desta revisão de literatura.

\section{HISTÓRICO}

A origem do nome parece ter vindo do tupi "cupa-yba" que significa "árvore de depósito" (Veiga Junior \& Pinto, 2002; Ramos, 2006) ou que tem jazida, referindo-se ao óleo que possui em seu interior (Veiga Junior \& Pinto, 2002).

O óleo de copaíba e suas propriedades medicinais eram muito conhecidos pelos índios latinoamericanos (Salvador, 1975; Cardim apud Cunha, 1998; Veiga Junior \& Pinto, 2002) que os utilizavam para curar feridas de guerreiros após batalhas (Maciel et al., 2002) e para passar no coto umbilical de recém nascidos (Maciel et al., 2002; Francisco, 2005).

Supõe-se que tal conhecimento advém da observação do comportamento de alguns animais feridos, que se atritavam no tronco das árvores de copaíba, na busca da cicatrização de suas feridas (Lloyd, 1898; Maciel et al., 2002; Veiga Junior \& Pinto, 2002; Francisco, 2005). Assim observou o holandês Gaspar Barléu (Barléu, 1974; Salvador, 1975; Veiga Junior \& Pinto, 2002).

\section{"Vêem-se estas plantas} esfoladas pelo atrito de animais, que procuram, instintivamente, este remédio da natureza".

Desta maneira desde os primeiros anos de descobrimento, com as observações de viajantes, freis jesuítas e historiadores, tal planta vem sendo indicada para diversos fins farmacológicos (Veiga Junior \& Pinto, 2002).

A primeira vez que o óleo de copaíba foi citado, provavelmente ocorreu no ano de 1534, quando foi publicada em Estrasburgo uma carta enviada por Pethus Martins ao Papa Leão X, em que a droga usada pelos índios nativos era chamada copei (Lloyd, 1898; Veiga Junior \& Pinto, 2002). Na mesma época, foi publicada, pelo jesuíta José Acosta, "De Natura Novi Orbi", traduzida para o francês em 1606 e que, em português, foi denominada "História natural e moral dos índios", que exacerbavam o bálsamo que, utilizado pelos índios, possuía excelente odor e muito maior efeito para curar feridas e outras enfermidades, prevendo inclusive o uso futuro da mesma pelos espanhóis (Acosta, 1792; Veiga Junior \& Pinto, 2002).

Em fins de 1560, o padre jesuíta José de Anchieta, em carta ao padre geral, comenta o poder cicatrizante potente do óleo de copaíba (Rodrigues, 1934; Veiga Junior \& Pinto, 2002; Veiga Junior et al., 2005).

Em meados do século XVI, em obra publicada sob o título "História da Província de Santa
Cruz, que vulgarmente chamamos de Brasil", Pero de Magalhães Gândavo foi o primeiro a descrever a eficácia do óleo de copaíba como analgésico e cicatrizante (Gandavo, 2004; Gurgel, 2004), que resultou em um comentário mundial a respeito da copaíba, tornando o óleo, junto com o cravo, anil e tabaco, um dos quatro produtos mais exportados pelas províncias do Maranhão e Grão Pará (Alencastro, 2000; Gurgel, 2004).

Em 1578, Jean Léry e posteriormente o padre Fernão Cardim, o padre Francisco Soares e Simão Travaços em 1584, 1594 e 1596, respectivamente, fizeram referência ao poder de cicatrização do óleo (Veiga Junior \& Pinto, 2002).

Em 1587, Gabriel Soares de Souza, na obra "Tratado descritivo do Brasil", registrou o uso do óleo pelos europeus e chamou nossos produtos naturais de "árvores e ervas da virtude" (Carrara Junior \& Meirelles, 1996; Veiga Junior \& Pinto, 2002).

A primeira descrição detalhada, explícita e ilustrada de florestas de copaíba, aconteceu no trabalho conjunto de MarcGrave e Piso, em 1648 (Lloyd, 1898), que descreveram aspectos morfológicos da planta que então recebeu como denominação o termo "copaíba" (Veiga Junior \& Pinto, 2002).

Em 1760, Jacquin descreveu em detalhes a primeira Copaifera, a Copaifera officinalis, porém, como essa não tinha frutos presentes, usou o modelo de MarcGrave e Piso, erroneamente (Veiga Junior \& Pinto, 2002). Finalmente, dois anos mais tarde, Cad von Linnaeus descreveu de forma correta a Copaifera officinalis, e então o gênero recebeu a descrição oficial de Copaifera L. (Lloyd, 1898).

Em 1818, o governo imperial do Brasil promulgou um ato regulando a extração das árvores que só poderiam ser derrubadas por conta do governo, vendidas com $20 \%$ de lucro, para a produção de mastros vergas de navio, com o objetivo de minimizar a destruição das florestas de copaíba que estava começando a acontecer, uma vez que a extração era feita sem critério (Veiga Junior \& Pinto, 2002).

No ano de 1821, o francês Desfontaines adicionou duas novas espécies ao gênero Copaifera: a C. guianensis e a C. langsdorffii (Lloyd, 1898). Quatro anos mais tarde, Hayne publicou uma monografia considerada confusa, que apresentava um total de oito espécies do Gênero em questão (Veiga Junior \& Pinto, 2002). O mesmo pesquisador, no ano seguinte, em estudo mais detalhado e expandido, apresentou 16 espécies de Copaifera com melhores descrições (Lloyd, 1898). As obras de Hayne serviram como ampla base de estudo para novas pesquisas, como a de Bertham, que, em expedição com o naturalista Von Martius em 1870, compilou "Flora Brasiliensis" (Veiga Junior \& Pinto, 2002).

Trabalhos mais recentes na descrição de 
outras novas espécies de Copaifera foram realizados por Harms e Ducke, que estudaram e descreveram espécies amazônicas, e cearenses em meados do século XX. Dwyer realizou descrição também de espécies americanas durante o mesmo período (Veiga Junior \& Pinto, 2002).

\section{Extração do óleo-resina}

O óleo de Copaíba provém de canais esquizolizígeos (Cascon, 2004), que são secretores, localizados em todas as partes da árvore. São canais formados pela dilatação de espaços intercelulares (meatos). O caráter mais saliente desse aparelho está no lenho (Cascon, 2004), onde os canais longitudinais, distribuídos em faixas concêntricas, nas camadas de crescimento demarcadas pelo parênquima terminal, reúnem-se com traçados irregulares em camadas lenhosas muitas vezes sem se comunicarem (Alencar, 1982). Há exudação das árvores de Copaifera (Maciel et al., 2002; Brito et al., 2005; Francisco, 2005; Lima Neto et al., 2008) em condições patológicas (Brito et al., 2005), sendo então o óleo, segundo Alencar (1982), produto da desintoxicação do organismo vegetal, e que funciona como defesa da planta contra animais, fungos e bactérias.

O óleo-resina pode ser obtido, através da perfuração no tronco da copaibeira (Lloyd, 1898; Veiga Junior \& Pinto, 2002; Cascon, 2004; Francisco, 2005;

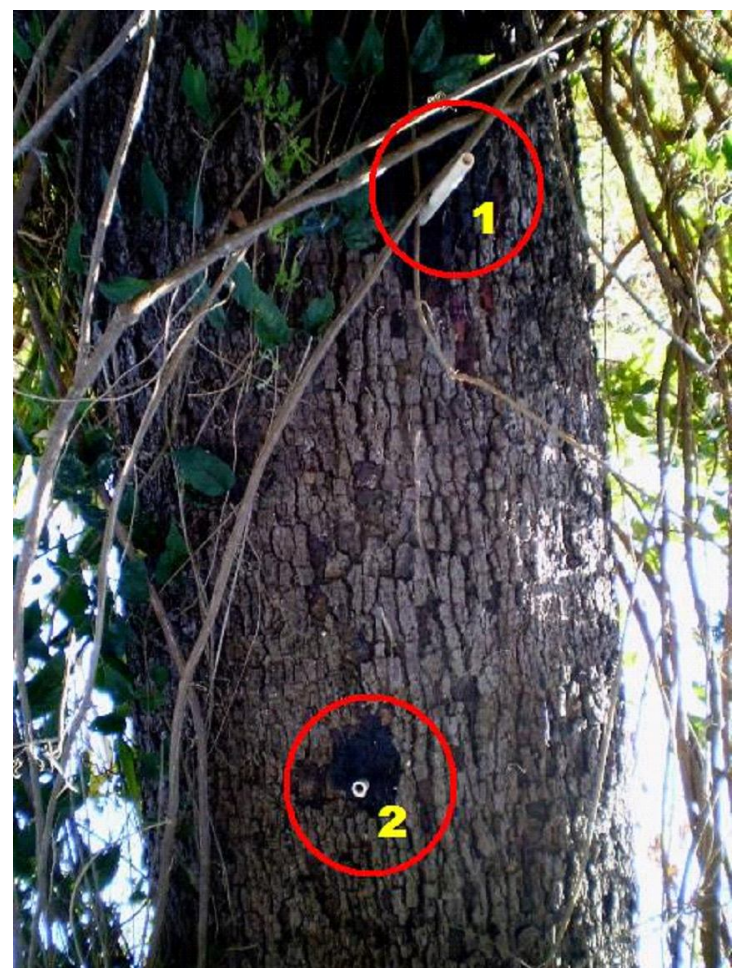

FIGURA 2. Extração do óleo de copaíba. O óleo escoa pelo orifício 2, quando ambos estão desobstruídos. Ao selar a abertura do orifício 1, cessa-se 0 escoamento do óleo.
Oliveira et al., 2005; Oliveira et al., 2006; Ramos, 2006; Rigamonte Azevedo et al., 2006) tendo sido utilizadas, desde os primórdios da exploração do produto, inúmeras formas de extração que provocavam danos sérios às árvores ou até provocavam sua morte (Matta, 1913). Porém, uma técnica tem sido considerada a única prática não agressiva, e é hoje a mais utilizada, consistindo na perfuração do tronco com um trado de aproximadamente 2 metros de diâmetro em dois furos. O primeiro deve ser feito 1 metro acima da base da planta e o segundo de 1 a 1,5 metros acima do primeiro (Alencar, 1982; Veiga Junior \& Pinto, 2002; Oliveira et al., 2006; Ramos, 2006; Rigamonte Azevedo et al., 2006). Insere-se então um cano de PVC de $3 / 4$ de polegada nos orifícios (Figura 2), por onde o óleo escoa, e reserva-se o óleo. Após a finalização da extração, veda-se o orifício para proteção contra fungos e cupins (Oliveira et al., 2006; Ramos, 2006; Rigamonte Azevedo et al., 2006) com o uso de argila (Ramos, 2006) ou tampa vedante de plástico (Oliveira et al., 2006) sendo ambas de fácil retirada para posteriores colheitas de óleo com maior facilidade de manipulação (Oliveira et al., 2006; Ramos, 2006).

Na primeira extração, a quantidade de óleoresina extraído varia bastante (Veiga Junior \& Pinto, 2002; Rigamonte Azevedo et al., 2004). A produção anual por árvore pode chegar, segundo alguns autores, a 50 litros/árvore (Matta, 1913; Grieve, 1995). A média de retirada de óleo, por vez para cada árvore, varia de 0,3 a 3 litros, dependendo da espécie e condições às quais está submetida, e algumas árvores podem chegar a fornecer até 30 litros em uma só retirada. Não há, porém, estudos definitivos sobre o tempo necessário para uma copaibeira recompor o óleo extraído. Sabese também que não se extrai óleo de todas as árvores de copaíba, mas não existem estudos precisos da média de árvores efetivamente fornecedoras de óleo, o que pode variar de acordo com as características do solo, clima, espécie da Copaifera e época seca ou chuvosa (Rigamonte Azevedo et al., 2004).

\section{Composição química do óleo-resina}

A primeira pessoa a descrever uma fração do óleo de copaíba foi Schweitzer em 1829 (Veiga Junior \& Pinto, 2002). Ele observou a cristalização de substância, a qual chamou de ácido copaívico, quando o óleo-resina permaneceu muito tempo em repouso. A partir daí outras substâncias foram sendo descritas, como o ácido oxycopaívico, por Fehling, em 1841, ácido metacopaívico, pelo pesquisador Strauss no ano de 1865. Tschirch e Keto, já no início do século $X X$, descreveram, além das duas últimas substâncias citadas anteriormente, também o ácido paracopaívico e o homocopaívico (Wood et al., 1940). Entretanto, de todos estes, apenas o ácido paracopaívico encontra semelhante na classificação atual (Veiga Junior \& Pinto, 2002). 
O óleo-resina de copaíba é uma substância natural (Cascon \& Gilbert, 2000) composta de uma parte sólida (Rigamonte Azevedo et al., 2004), resinosa não volátil (Lloyd, 1898) formada por ácidos diterpênicos (Cascon \& Gilbert, 2000; Rigamonte Azevedo et al., 2004; Veiga Junior et al., 2005; Oliveira et al., 2006; Ramos, 2006) responsável por 55 a 60 $\%$ do óleo (Rigamonte Azevedo et al., 2004), que, segundo Freire et al. (2006), é utilizada como bálsamos, diluída na outra parte, um óleo essencial (Lloyd, 1898; Cascon \& Gilbert, 2000; Rigamonte Azevedo et al., 2004), composto de sesquiterpenos (Rigamonte Azevedo et al., 2004; Araújo Júnior et al., 2005; Veiga Junior et al., 2005; Oliveira, et al., 2006; Ramos, 2006). Estes podem ser divididos em sesquiterpenos oxigenados (álcoois) e hidrocarbonetos sesquiterpênicos (Cascon \& Gilbert, 2000) que, segundo Maciel et al. (2002), possuem maior atividade antiinflamatória quando comparados aos outros dois grupos presentes.

O óleo extraído pode variar em relação à concentração e natureza dos diterpenos e sesquiterpenos presentes (Veiga Junior et al., 2005; Ramos, 2006) de acordo com variações de espécies, fatores biológicos como insetos e fungos (Ramos, 2006), ou fatores abióticos (Oliveira et al., 2006; Ramos, 2006), embora alguns estudos com o uso da Copaifera duckei não tenham apresentado diferença significativa na composição de óleos extraídos das mesmas árvores, em épocas sazonais diferentes (Cascon \& Gilbert, 2000).

Apesar de poderem apresentar alterações nas quantidades e tipos de diterpenos e sesquiterpenos, apenas essas classes de substâncias podem estar presentes na composição do óleo puro (Veiga Junior et al., 2005).

Segundo alguns autores, existem descritos como provenientes de algum óleo de copaíba, 72 sesquiterpenos e 27 ou 28 diterpenos, dependendo da fonte (Veiga Junior \& Pinto, 2002; Rigamonte Azevedo et al., 2004; Pacheco et al., 2006), embora, segundo Veiga Junior \& Pinto (2002), apenas 5 espécies de copaífera tiveram a composição descrita na literatura (C. multijuga Hayne, C. langsdorffii, $C$. cearensis, C. officinalis L. e C. reticulada Ducke), totalizando apenas 17 relatos químicos no total (Lima Neto et al., 2008).

Os principais sesquiterpenos encontrados no óleo-resina da copaíba são $\beta$-cariophileno (Craveiro et al., 1981), que possui comprovada ação antiinflamatória, antibacteriana, antifúngica e antiedêmica (Veiga Junior \& Pinto, 2002; Oliveira et al., 2006; Ramos, 2006), a $\beta$-bisaboleno (Maciel et al., 2002; Veiga Junior \& Pinto, 2002; Oliveira et al., 2006; Ramos, 2006), com propriedades descritas como antiinflamatórias e analgésicas (Oliveira et al., 2006; Ramos, 2006), além do $\alpha$-humuleno, $\alpha$ e $\beta$ - selineno (Veiga Junior \& Pinto, 2002; Oliveira et al., 2006; Ramos, 2006), $\alpha$-bisabolol, $\beta$ - elemeno (Maciel et al., 2002; Veiga Junior \& Pinto, 2002), $\gamma$-cadineno (Maciel et al., 2002; Veiga Junior \& Pinto, 2002; Silva et al., 2006), $\alpha$-cadinol (Veiga Junior \& Pinto, 2002), entre muitos outros. Segundo Silva et al. (2006), o óleo essencial extraído das folhas da copaibeira possui composição semelhante à da parte volátil sesquiterpênica do óleo-resina, com substâncias como $\beta$-cariophileno, cadinol, Germacreno D e B e $\gamma$ cadineno.

A parte resinosa do óleo-resina é composta pelos seguintes diterpenos: ácido hardwíckico, colavenol (Maciel et al., 2002; Veiga Junior \& Pinto, 2002), ácido copaiférico ou copaífero (Veiga Junior \& Pinto, 2002; Araújo Júnior et al., 2005), ácido copaiferólico, ácido calavênico, ácido patagônico, ácido copálico entre outros. O ácido copálico é usado como marcador do óleo da copaibeira, tendo em vista o fato de que foi o único encontrado em todos os óleos analisados por cromatografia gasosa para identificação da composição dos mesmos (Veiga Junior \& Pinto, 2002).

\section{Aplicações industriais do óleo de copaíba}

O óleo de copaíba tem sido utilizado extensivamente, com diversas funções. Em alguns lugares do norte do Brasil, caboclos fazem uso do mesmo, como combustível na iluminação pública (Veiga Junior \& Pinto, 2002). Pelo fato de ser fonte muito rica e renovável de hidrocarbonetos, o óleoresina tem sido intensamente avaliado como uma fonte de combustível, tendo sucesso comprovado, quando em mistura na proporção 1:9 com o óleo diesel (Calvin, 1983).

$\mathrm{Na}$ indústria de perfumes, o óleo essencial de copaíba é muito utilizado (Veiga Junior \& Pinto, 2002; Cascon, 2004; Pacheco et al., 2006; Ramos, 2006; Rigamonte Azevedo et al., 2006) como um excelente fixador de odores, combinando perfeitamente suas notas frescas e acres com essências portadoras de notas florais (Veiga Junior \& Pinto, 2002).

Outros usos bastante difundidos do óleo da copaibeira são: secativo (Veiga Junior \& Pinto, 2002) na indústria de vernizes (Cascon, 2004; Ramos, 2006; Rigamonte Azevedo et al., 2006), solventes em pinturas de porcelanas (Veiga Junior \& Pinto, 2002), aditivo na confecção de borracha sintética (Tillotson, 1945), aditivos de alimentos com aprovação pelo FDA (Food and Drugs Administration) (Cascon, 2004), na indústria de cosméticos (Veiga Junior \& Pinto, 2002; Cascon, 2004; Pacheco et al., 2006; Ramos, 2006) pelas suas propriedades emolientes, bactericidas e antiinflamatórias, na fabricação de cremes, sabonetes, xampus e amaciantes de cabelos (Veiga Junior \& Pinto, 2002; Cascon, 2004). 
A madeira extraída da copaibeira tem sido comercializada com bastante freqüência (Veiga Junior \& Pinto, 2002; Cascon, 2004; Freire et al., 2006) pelas suas propriedades desejáveis ao uso em marcenaria (Loureiro, 1979), carpintaria (Cascon, 2004), sendo também utilizadas na construção civil e naval (Carvalho, 1994; Cascon, 2004), além da fabricação de carvão (Veiga Junior \& Pinto, 2002).

Além de todas as aplicações supracitadas, o óleo de copaíba ainda apresenta uma gama enorme de utilizações na medicina popular, indígena e na indústria farmacêutica (Ming, 1995; Barata \& Mendonça, 1997; Shanley et al., 1998; Leite et al., 2001; Rigamonte Azevedo et al., 2004).

\section{Utilização medicinal do óleo de copaíba}

O óleo-resina extraído da copaibeira recebe indicação da medicina tradicional, para inúmeras finalidades, das mais diferentes naturezas, e tem sido há vários anos matéria de vários estudos, visando comprová-las ou adaptá-las a novas terapias. No ano de 1972, o Food and Drug Administration, órgão americano regulamentador de drogas, aprovou o óleo de copaíba, após ser submetido a testes de sensibilização e irritação, com o uso de 25 voluntários, obtendo-se resultado negativo para ambos (Veiga Junior \& Pinto, 2002). As principais propriedades terapêuticas citadas são: a atividade antiinflamatória (Vieira, 1992; Maciel et al., 2002; Rigamonte Azevedo et al., 2004; Araújo Júnior et al., 2005; Brito et al., 2005; Francisco, 2005; Freire et al., 2006; Pacheco et al., 2006; Ramos, 2006; Silva et al., 2006), que tem como principais componentes responsáveis os hidrocarbonetos, sesquiterpênicos, especialmente o $\beta$-bisaboleno e $\beta$-cariophileno (Veiga Junior \& Pinto, 2002; Oliveira et al., 2005; Veiga Junior et al., 2005; Ramos, 2006), ação cicatrizante (Brito et al., 2000; Maciel et al., 2002; Veiga Junior \& Pinto, 2002; Rigamonte Azevedo et al., 2004; Araújo Júnior et al., 2005; Brito et al., 2005; Francisco, 2005; Veiga Junior et al., 2005; Ramos, 2006; Silva et al., 2006), potencial anti-séptico (Goodman \& Gilman, 1945; Robbers et al., 1996; Maciel et al., 2002; Veiga Junior \& Pinto, 2002; Cascon, 2004; Rigamonte Azevedo et al., 2004; Veiga Junior et al., 2005; Ramos, 2006), antitumoral (Rodrigues, 1989; Maciel et al., 2002; Veiga Junior \& Pinto, 2002; Rigamonte Azevedo et al. 2004; Araújo Junior et al., 2005; Francisco, 2005; Veiga Junior et al., 2005; Freire et al., 2006; Oliveira et al, 2006; Pacheco et al., 2006; Silva et al., 2006), antibacteriano (Veiga Junior \& Pinto, 2002; Drumond et al., 2004; Gonçalves et al., 2005; Veiga Junior et al., 2005; Freire et al., 2006; Pieri, 2007), germicida (Bloise, 2003), expectorante (Brito et al., 2000; Maciel, et al., 2002; Rigamonte Azevedo et al., 2004; Brito et al., 2005; Freire et al., 2006; Ramos, 2006), diurético (Maciel et al., 2002; Freire et al., 2006; Silva et al.,
2006) e analgésico (Veiga Junior \& Pinto, 2002; Gurgel, 2004; Oliveira et al., 2005; Pacheco et al., 2006).

Outras indicações também são conhecidas e citadas, como a ação antiviral (Veiga Junior \& Pinto, 2002; Gurgel, 2004), antidiarréica, contra o reumatismo, psoríase (Brito et al., 2000; Maciel et al., 2002), hemorragias, moléstias de pele, urticárias (Maciel et al., 2002), pneumonia, eczema, paralisia, cefaléia, picada de cobra (Rigamonte Azevedo et al., 2004), sífilis (Pacheco et al., 2006), leishmaniose, como antitetânico, antiblenorrágico (Rigamonte Azevedo et al., 2004), antileucorréico e cercaricida (Maciel et al., 2002), além de ações antiulcerogênicas, estimulantes (Ramos, 2006) e afrodisíacos (Ribeiro, 1971). O extrato das folhas da copaibeira tem tido apontado como uma de suas funções, a atividade antioxidante, função não comprovada no óleo-resina (Veiga Junior \& Pinto, 2002; Rigamonte Azevedo et al., 2004; Araújo Júnior et al., 2005; Ramos, 2006)

Outras ações têm sido descobertas, como a de vasorelaxante, citotóxico e embriotóxico (Costa Lotufo et al., 2002), com o isolamento do diterpeno, ácido caurenóico, encontrado até hoje apenas na Copaifera langsdorffii (Veiga Junior et al., 2005) e que acumula também atividades antiinflamatória e protetora de colite induzida por ácido acético (Paiva et al., 2004; Veiga Junior et al., 2005). Além disso, pesquisas realizadas nas últimas duas décadas apontam o óleo de copaíba para o campo da Odontologia, como substituto do eugenol em três formulações (Costa et al., 1996; Veiga Junior \& Pinto, 2002; Cascon, 2004; Ramos, 2006) : Na mistura com o óxido de zinco, na formulação do cimento endodôntico, para obturações de canais radiculares, com vantagens sobre a formulação usual, por produzir menor irritação (Ribeiro, 1989). Em composição obturadora provisória medicamentosa, em combinação com o hidróxido de cálcio, apresenta melhor capacidade antimicrobiana, em relação à formulação com o eugenol (Veiga Junior \& Pinto, 2002; Ramos, 2006). A pasta feita com o óleo de copaíba apresentou pequeno potencial irritativo, sendo preferível ao médio potencial do eugenol, além de potencializar as propriedades do $\mathrm{CaOH}$, na compatibilidade tecidual, na indução da formação de tecido mineralizado; em cimento formado com a união de óleo de copaíba, $\mathrm{CaOH}$ e óxido de zinco mostra maior eficiência nas infiltrações apicais em obturações de canais radiculares (Ramos, 2006).

Recentemente testes têm também sido realizados em ensaios laboratoriais com a finalidade de comprovar ação antimicrobiana da copaíba sobre bactérias formadoras da placa dental (Pieri, 2007; Valdevite et al., 2007), tendo inclusive sido registrado um gel à base de copaíba, para esta finalidade (Simões, 2004). A utilização do óleo de copaíba sobre as bactérias formadoras de placa dental foi 
comprovada recentemente em estudo realizado em cães, tendo sido obtido ao final do período experimental, redução significativa da formação da placa dental nos animais com a utilização de solução a base do fitofármaco (Pieri, 2007). Pieri (2007) também testou in vitro a atividade antimicrobiana do óleo de copaíba, sobre a bactéria Streptococcus pyogenes, causadora de inflamações de garganta, obtendo resultados positivos na inibição do crescimento deste microrganismo.

\section{CONCLUSÃO}

O óleo de copaíba tem sido utilizado por mais de 500 anos na medicina tradicional popular, com uma grande diversidade de aplicações. Alguns trabalhos têm sido realizados com o objetivo de estabelecer um método de extração do óleo não prejudicial à planta e determinar a composição deste. Algumas outras pesquisas tem se direcionado para a comprovação das atividades medicinais atribuídas ao óleo e a indicação científica deste à terapêutica de várias doenças. Porém, levando em consideração as diversas indicações, estes estudos têm-se apresentado ainda restritos e escassos. Muitos outros devem ainda ser direcionados às importantes atividades descritas, tais como antitumoral, antiinflamatória, antimicrobiana contra uma grande diversidade de microrganismos e cicatrizante sobre diferentes tecidos do corpo. Ainda aponta-se também a possibilidade de estudar as atividades das diferentes frações componentes do óleo, o que se reverteria na criação de alternativas terapêuticas para muitas patologias humanas e animais.

\section{REFERÊNCIA}

ACOSTA, J. História nature y moral de las índias: Libro tercero. Madrid: Cervantes Virtual, 1792. 253p.

ALENCAR, J. Estudos silviculturais de uma população natural de Copaifera multijuga Hayne - Leguminosae, na Amazônia central. 2 - produção de óleo resina. Acta Amazônica, v.12, n.1, p.79-82, 1982.

ALENCASTRO, L.F. $O$ trato dos viventes, formação do Brasil no Atlântico sul. Séculos XVI e XVII. São Paulo: Companhia das Letras, 2000. 525p.

ARAÚJO JÚNIOR, F.A. et al. Efeito do óleo de copaíba nas aminotransferases de ratos submetidos à isquemia e reperfusão hepática com e sem pré-condicionamento isquêmico. Acta Cirúrgica Brasileira, v.20, n.1, p.93-9, 2005.

BARATA, L.E.S.; MENDONÇA, C. Copaíba: propriedades farmacológicas, etnofarmacológicas, usos. Rio de Janeiro: GEF/Instituto pró-natura, 1997. 31p. (Relatório1) BARLÉU, G. Historia dos feitos recentemente praticados durante oito anos no Brasil (Coleção reconquista do Brasil, v.15). Belo Horizonte: Ed. Itatiaia, 1974. 141p.
BLOISE, M.I. Óleos vegetais e especialidades da floresta amazônica. Cosmetics \& Toiletries, v.15, n.5, p. 46-9, 2003.

BRITO, M.V.H. et al. Copaiba oil effect on urea and creatinine serum levels in rats submitted to kidney ischemia and reperfusion syndrome. Acta Cirúgica Brasileira, v.20, n.3, p.243-6, 2005.

BRITO, N.M.B. et al. Aspectos morfológicos e morfométricos do colo uterino de ratas ooforectomizadas após aplicação de óleo de copaíba. Revista Brasileira de Ginecologia e Obstetrícia, v.22, n.8, p.489-93, 2000. CALVIN, M. New sources for fuel and materials. Science, v.219, p.24-6, 1983.

CARDIM, F. Do clima e terra do Brasil, 1584, p.21 apud CUNHA, A.G. Dicionário histórico das palavras portuguesas de origem Tupi. 4.ed. Brasília: Companhia Melhoramentos, 1998. 112p.

CARRARA JÚNIOR, E.; MEIRELLES, H. A indústria química e o desenvolvimento do Brasil - 1500-1889. São Paulo: Metalivros, 1996. 115p.

CARVALHO, J.B.M. O Norte e a indústria de óleos vegetais sob o aspecto técnico-econômico. Rio de Janeiro: Ministério da Agricultura, 1942. 135p.

CARVALHO, P.E.R. Espécies florestais brasileiras: recomendações silviculturais, potencialidades e uso de madeira. Brasília: EMBRAPA/CNPF, 1994. 640p.

CASCON, V.; GILBERT, B. Characterization of the chemical composition of oleoresins of Copaifera guianensis Desf., Copaifera duckei Dwyer and Copaifera multijuga Hayne. Phytochemistry, v.55, n.7, p.773-8, 2000. CASCON, V. Copaíba - Copaifera spp. In: CARVALHO, J.C.T. Fitoterápicos antiinflamatórios: aspectos químicos, farmacológicos e aplicações terapêuticas. Ribeirão Preto: Tecmedd, 2004. 480p.

COSTA, C.A.S. et al. Estudo preliminar de compatibilidade biológica dos cimentos de óxido de zinco e copaíba e óxido de zinco e eugenol: avaliação histológica de implantes subcutâneos em ratos. Revista de Odontologia UNESP, v.25, n.1, p.19-26, 1996.

COSTA-LOTUFO, L.V. et al. The cytotoxic and embryotoxic effects of kaurenoic acid, a diterpene isolated from Copaifera langsdorffii oleo-resin. Toxicon, v.40, n.8, p.1231-4, 2002.

CRAVEIRO, A.A. et al. Óleos essenciais de plantas do Nordeste. Fortaleza: Editora UFC, 1981. 150p.

DRUMOND, M.R.S. et al. Comparative study in vitro of the antibacterial activity from phytotherapeutic products against cariogenical bactérias. Pesquisa Brasileira em Odontopediatria e Clinica Integrada, v.4, n.1, p.33-8, 2004. FOOD AND DRUGS ADMINISTRATION. Food chemical codex. 2.ed. Washington: National Academic Press, 1972. 218p.

FRANCISCO, S.G. Uso do óleo de copaíba (Copaifera officinalis) em inflamação ginecológica. Femina, v.33, n.2, p.89-93, 2005.

FREIRE, D.B. et al. Efeito dos óleos vegetais de andiroba (Carapa sp.) e copaíba (Copaifera sp.) sobre forídeos, pragas de colméias, (Díptera: Phoridae) na Amazônia central. Acta Amazônica, v.36, n.3, p.365-8, 2006.

GANDAVO, P.V.M. A Primeira história do Brasil- história da província de Santa Cruz a que vulgarmente chamamos de Brasil. Modernização do texto original de 1576 e notas: Sheila Moura Hue e Ronaldo Menegaz. 
Rio de Janeiro: Jorge Zahar Editor, 2004. p.75-89. GOODMAN, L.; GILMAN, A. As bases farmacológicas da terapêutica. Rio de Janeiro: Editora Guanabara, 1945. v.2, $1461 \mathrm{p}$.

GONÇALVES, A.L. et al. Estudo comparativo da atividade antimicrobiana de extratos de algumas árvores nativas. Arquivos do Instituto de Biologia, v.72, n.3, p.353-8, 2005. GRIEVE, M. A modern herbal. London: Tiger books international, 1995. 221p.

GURGEL, C.B.F.M. A fitoterapia indígena do Brasil colonial (os primeiros dois séculos). In: ENCONTRO REGIONAL DE HISTÓRIA, 11., 2004. Anais eletrônicos... Rio de Janeiro: UFF, 2004. Disponível em: <http:// www.uff.br/ichf/anpuhrio/Anais>. Acesso em: 24 fev. 2007. INDEX KEWENSIS. Supplement XX. Oxford: Claredon Press, 1996. 346p.

LEITE, A.C. et al. Recomendações para o manejo sustentável do óleo de copaíba. Rio Branco: UFAC/ SEFE, 2001. 38p.

LIMA NETO, J.S.; GRAMOSA, N.V.; SILVEIRA, E.R. Constituintes químicos do fruto de Copaifera langsdorffii Desf. Química Nova, v.31, n.5, p.1078-80, 2008.

LLOYD, J.U. Copaifera officinalis. Chicago: The Western Druggist,1898. 13p.

LORENZI, H. Árvores brasileiras: manual de identificação e cultivo de plantas arbóreas nativas do Brasil. Nova Odessa: Instituto Plantarum, 1992. 382p. LOUREIRO, A. Essências madeireiras da Amazônia, Manaus: INPA/CNPq, 1979. v.1, 125p.

MACIEL, M.A. et al. Plantas medicinais: a necessidade de estudos multidiciplinares. Quimica Nova, v.25, n.3, p.429-38, 2002.

MATTA, A.A. Flora medica braziliense. Manaus: Imprensa Oficial, 1913. 318p.

MING, L.C. Levantamento de plantas medicinais na reserva extrativista "Chico Mendes" Acre. 1995. 268p. Tese (Doutorado) - Universidade Estadual Paulista, Instituto de Biociências, Botucatu.

MORS, W.; RIZZINI, C.T. Useful plants of Brazil. San Francisco: Holden-Day Inc., 1966. 45p.

OLIVEIRA, E.C.P. et al. Identificação da época de coleta do óleo-resina de copaíba (Copaifera spp.) no município de Moju-PA. Revista Brasileira de Plantas medicinais, v.8, n.3, p.14-23, 2006.

OLIVEIRA, U.D. et al. Avaliação do ciclo celular de Aspergillus nidulans exposto ao extrato da planta Copaifera officinalis L. Revista Saúde e Biologia, v.1, n.2, p.42-7, 2005.

PACHECO, T.A.R.C. et al. Antimicrobial activity of copaíba (Copaifera spp) balsams. Revista Brasileira de Plantas Medicinais, v.8, p.123-4, 2006.

PAIVA, L.A.F. et al. Protective effect of Copaifera langsdorffii oleo-resin against acetic acid-induced colitis in rats. Journal of Ethnopharmacology, v.93, n.1, p.516, 2004.

PIERI, F.A. Efeito (in vitro/ in vivo) do óleo de copaíba (Copaifera officinalis) sobre bactérias formadoras de placa dental em cães (Canis lupus Familiaris). 2007. 85p. Dissertação (Mestrado em Ciência Animal) Universidade José do Rosário Vellano, Alfenas. RAMOS, M.F.S. Desenvolvimento de microcápsulas contendo a fracão volátil de copaíba por spray-drying: estudo de estabilidade e avaliação farmacológica. 2006 . 132p. Tese (Doutorado em Ciências Farmacêuticas) Universidade de São Paulo, Ribeirão Preto.

RIBEIRO, A. Avaliação do potencial irritativo do óleoresina de copaíba (substituto do eugenol) na fase exsudativa do processo inflamatório. 1989. 77p. Tese (Doutorado) - Faculdade de Odontologia, Universidade de São Paulo, Bauru.

RIBEIRO, L. Medicina no Brasil colonial. Rio de Janeiro: Editora Sul Americana, 1971. 211p.

RIGAMONTE AZEVEDO, O.C. et al. Potencial de produção de óleo-resina de copaíba (Copaifera sp.) de populações naturais do sudoeste da Amazônia. Revista Árvore, v.30, n.4, p.583-91, 2006.

RIGAMONTE AZEVEDO, O.C. et al. Copaíba: ecologia e produção de óleo-resina. Rio Branco: EMBRAPA, MAPA, 2004. 28p.

ROBBERS, J.E. et al. Pharmacognosy and pharmacobiotechnology. Baltimore: Williams \& Wilkins, 1996. 100p.

RODRIGUES, L. Anchieta e a medicina. Belo Horizonte: Edições Apollo, 1934. 361p.

RODRIGUES, R.M. A flora da Amazônia. Belém: Editora Cejup, 1989. 463p.

SALVADOR, V. História do Brasil: 1500-1627. 6.ed. São Paulo: Melhoramentos, 1975. 63p.

SHANLEY, P. et al. Frutíferas da mata na vida amazônica. Belém: Editora Supercores, 1998. 127p.

SILVA, F.H. et al. Estudo do óleo essencial e extrato hidrometanólico de Copaifera langsdorffii Desf (Caesalpinaceae) do cerrado e mata atlântica. In: REUNIÃO NACIONAL DA SOCIEDADE BRASILEIRA DE QUÍMICA, 29., 2006. Águas de Lindóia. Anais eletrônicos... São Paulo: Instituto de Química da USP, 2006. Disponível em: <http://www.sbq.org.br/29ra>. Acesso em: 24 fev. 2007. SIMÕES, C.A.C.G. Patente Brasileira no PI0404266-2. Rio de Janeiro: Instituto Nacional de Propriedade Industrial, 2004.

TILLOTSON, N.E. U.S. patent 2.379.389. 1945.

VALDEVITE, L.M. et al. Study of the in vitro effect of copaíba oil upon virulence factors of the cariogenic bacterium Streptococcus mutans. In: IUBMB CONFERENCE, 36., REUNIÃO ANUAL DA SBBQ, 10., 2007. Salvador. Anais... São Paulo: Sociedade Brasileira de Bioquímica e Biologia Molecular, 2007.

VAN DEN BERG, M.E. Plantas medicinais da Amazônia: Contribuição ao seu conhecimento sistemático Brasília: CNPq-MPEG, 1982. 145p.

VEIGA JUNIOR, V.F.; PINTO, A.C. O Gênero Copaifera L. Química nova, v.25, n.2, p.273-86, 2002.

VEIGA JUNIOR, V.F. et al. Plantas medicinais: cura segura? Química nova, v.28, n.3, p.519-28, 2005.

VIEIRA, L.S. Fitoterapia da Amazônia. 2.ed. São Paulo: Editora Agronômica Ceres, 1992. 347p.

WOOD, G. et al. The dispensatory of the United States of America. 22.ed. Londres: J.B. Lippincott Company, 1940. 369p.

XENA, N.;BERRY, P.E. Copaifera L. In: STEYERMARK, J.A. et al. Flora of the Venezuelan Guyana. Missouri: Botanical Garden Press, 1998. p.45-7. 